\title{
DERMATITE DE CONTACTO ALÉRGICA PROFISSIONAL AO MERCAPTOBENZOTIAZOL
}

\author{
Catarina Araúio', C. Resende, T. Pereira², José Carlos Fernandes ${ }^{3}$, Celeste Brito ${ }^{4}$ \\ 'Interna do Internato Complementar de Dermatologia e Venereologia/Resident, Dermatology and Venereology \\ ${ }^{2}$ Assistente Hospitalar de Dermatologia e Venereologia/Consultant, Dermatology and Venereology. \\ ${ }^{3}$ Assistente Hospitalar Graduado(a) de Dermatologia e Venereologia/Graduated Consultant, Dermatology and \\ Venereology \\ ${ }^{4}$ Chefe de Serviço, Diretora do Serviço de Dermatologia e Venereologia/Consultant Chief, Head of Department of \\ Dermatology and Venereology, Hospital de Braga, Braga, Portugal
}

RESUMO - Introdução: A dermatite de contacto alérgica ao mercaptobenzotiazol e seus derivados tem sido reconhecida de forma crescente nos países industrializados devido ao seu uso alargado na polimerização da borracha sintética. Os autores descrevem dois casos clínicos com relevante exposição profissional. Caso 1: Homem de 46 anos, trabalhador na construção civil, com uma dermatite de oito meses de evolução, pruriginosa, localizada apenas nos punhos, refratária a corticóide tópico de alta potência e anti-histamínico oral. Ao exame objetivo apresentava placas eritemato-violáceas infiltradas e pápulas eritematosas escoriadas em ambos os punhos. Os testes Prick e RAST ao latex foram negativos. Foram efetuados testes epicutâneos com a bateria padrão do Grupo Português de Estudo de Dermatite de Contacto, bateria de borrachas, bateria de colas e fragmentos da própria luva tendo-se verificado positividade ao mercaptobenzotiazol $(++)$, mistura de mercapto $(++)$ e à faixa elástica presente no punho das luvas, às $72 \mathrm{~h}$ e aos 7 dias. Caso 2: Homem de 61 anos, carpinteiro, com placas eritematosas infiltradas, pruriginosas, na região geniana e extensão para região cervical posterior, com cerca de 12 meses de evolução. Foram realizados testes epicutâneos com a bateria padrão do Grupo Português de Estudo de Dermatite de Contacto, bateria de borrachas e colas, bateria de cosméticos e fragmentos da própria máscara de proteção. Verificou-se positividade para mercaptobenzotiazol $(++)$, mistura mercapto $(++)$ e elástico de máscara $(++)$ na leitura das 72 horas e aos 7 dias. Discussão: Apesar de serem alergénios bem conhecidos, o mercaptobenzotiazol e seus derivados podem estar presentes noutras fontes de exposição à borracha como o tecido elástico no vestuário. Uma vez o trabalhador sensibilizado, pode ser causa de incapacidade quer para a profissão quer para as atividades de vida diária.

PALAVRAS-CHAVE - Benzotiazóis; Dermatite de contacto alérgica; Dermatite ocupacional.

\section{OCCUPATIONAL ALLERGIC CONTACT DERMATITIS TO MERCAPTOBENZOTIAZOL}

ABSTRACT - Introduction: Allergic contact dermatitis to mercaptobenzothiazole and its derivatives has been increasingly recognized in industrialized countries because of their widespread use in polymerization of synthetic rubber. The authors describe two clinical cases with relevant occupational exposure. Case reports: A 46 year-old male patient, with an eight month pruritic dermatitis, limited to the wrists, refractory to high potency topical corticosteroid and oral antihistamine. Physical examination showed erythematous-violaceous infiltrated plaques and excoriated erythematous papules on both wrists. Prick tests and RAST to latex were negative. Epicutaneous tests were performed with basic series from Portuguese Contact Dermatitis Group, rubber and glue series and fragments of the sleeve and it was found positivity for mercaptobenzothiazole $(++)$, mercapto mix $(++)$ and elastic band of gloves in reading at $72 \mathrm{~h}$ and at 7 days. A 61 years old non atopic man, woodworker, with a 12- month history of itching, erythema, infiltration of genius region of face and extension to posterior cervical region. Epicutaneous tests were performed with basic series from Portuguese Contact Dermatitis Group, rubber and glve series, cosmetics series and fragments of the protection mask work and it was found positivity for mercaptobenzothiazole $(++)$, mercapto mix $(++)$ and elastic band of mask $(++)$ in reading at $72 \mathrm{~h}$ and at 7 days. Discussion: Despite being well known allergens, mercaptobenzothiazole and 
its derivatives may be present in other sources of exposure to rubber like elastic fabric in clothing. Once the worker sensitized, it can be an important cause of disability in an occupational and personnal setting.

KEY-WORDS - Benzothiazoles; Dermatitis, Allergic contact; Dermatitis, occupational.

\author{
Conflitos de interesse: Os autores declaram não possuir conflitos de interesse. \\ No conflicts of interest. \\ Suporte financeiro: $O$ presente trabalho não foi suportado por nenhum subsídio ou bolsa. \\ No sponsorship or scholarship granted. \\ Direito à privacidade e consentimento escrito / Privacy policy and informed consent: Os autores declaram \\ que pediram consentimento ao doente para usar as imagens no artigo. The authors declare that the patient gave \\ written informed consent for the use of its photos in this article.
}

Recebido/Received - Agosto/August 2014; Aceite/Accepted - Setembro/September 2014

\title{
Correspondêncią: \\ Dr. . Catarina Araújo
}

Serviço de Dermatologia e Venereologia

Hospital de Braga, Sete Fontes - São Victor

4710-243 Braga, Portugal

Tel: +351-253-027-000

Fax: +351-253-027-999

E-mail: catarina.portela.aravjo@gmail.com

\section{INTRODUÇÃO}

A dermatite de contacto alérgica (DCA) ao mercaptobenzotiazol e seus compostos tem sido reconhecida de forma crescente nos países industrializados devido ao uso alargado da borracha sintética.

Na literatura estão descritos múltiplos casos de dermatite de contacto alérgica ao mercaptobenzotiazol (2-Mercaptobenzotiazol) e seus derivados, morfolinil mercaptobenzotiazol, $\mathrm{N}$-ciclohexil-2-benzotiazol-sulfenamida e 2,2' benzotiazol dissulfeto, com potenciais reações cruzadas entre si, via oxidação-redução'. São frequentes as reações alérgicas de tipo IV, de hipersensibilidade tipo retardada, sendo raras as reações de tipo I (classificação Gel e Coomb's). No entanto, nos últimos 30 anos tem-se observado um declínio da prevalência de DCA ao mercaptobenzotiazol ${ }^{2,3}$.

Descrevem-se dois doentes com dermatite de contacto alérgica profissional ao mercaptobenzotiazol.

\section{Caso 1}

Doente do sexo masculino, 46 anos, trabalhador na construção civil, com dermatite crónica, pruriginosa, localizada apenas nos punhos, com oito meses de evolução, refratária a corticóide tópico de alta potência e anti-histamínico oral. Ao exame objetivo apresentava placas eritemato-violáceas infiltradas e pápulas eritematosas escoriadas em ambos os punhos (Fig.s la e 1b). Sem história pessoal ou familiar de atopia ou patologia cutânea.

Os testes Prick e RAST ao latex foram negativos. Foram efetuados testes epicutâneos com a bateria padrão do Grupo Português de Estudo de Dermatite de Contacto (GPEDC), bateria de borrachas e colas e fragmentos da própria luva tendo-se verificado positividade ao mercaptobenzotiazol $(++)$, mistura de mercapto $(++)$ (Fig. 1c) e com a faixa elástica presente no punho das luvas, na leitura das $72 \mathrm{~h}$ e aos 7 dias (Fig. 1d). Os restantes alergénios testados foram negativos incluindo os resultados com o restante corpo da luva (interior e exterior).

\section{Caso 2}

Homem de 61 anos, carpinteiro, com placas eritematosas, pruriginosas e infiltradas, na região geniana e com extensão para região cervical posterior, com evolução de cerca de 12 meses (Fig.s $2 a$ e $2 b$ ) clinicamente interpretado como lúpus eritematoso discoide. As lesões cutâneas eram refratárias a corticóide tópico de alta potência e anti-histamínico oral. Foi 
cinec
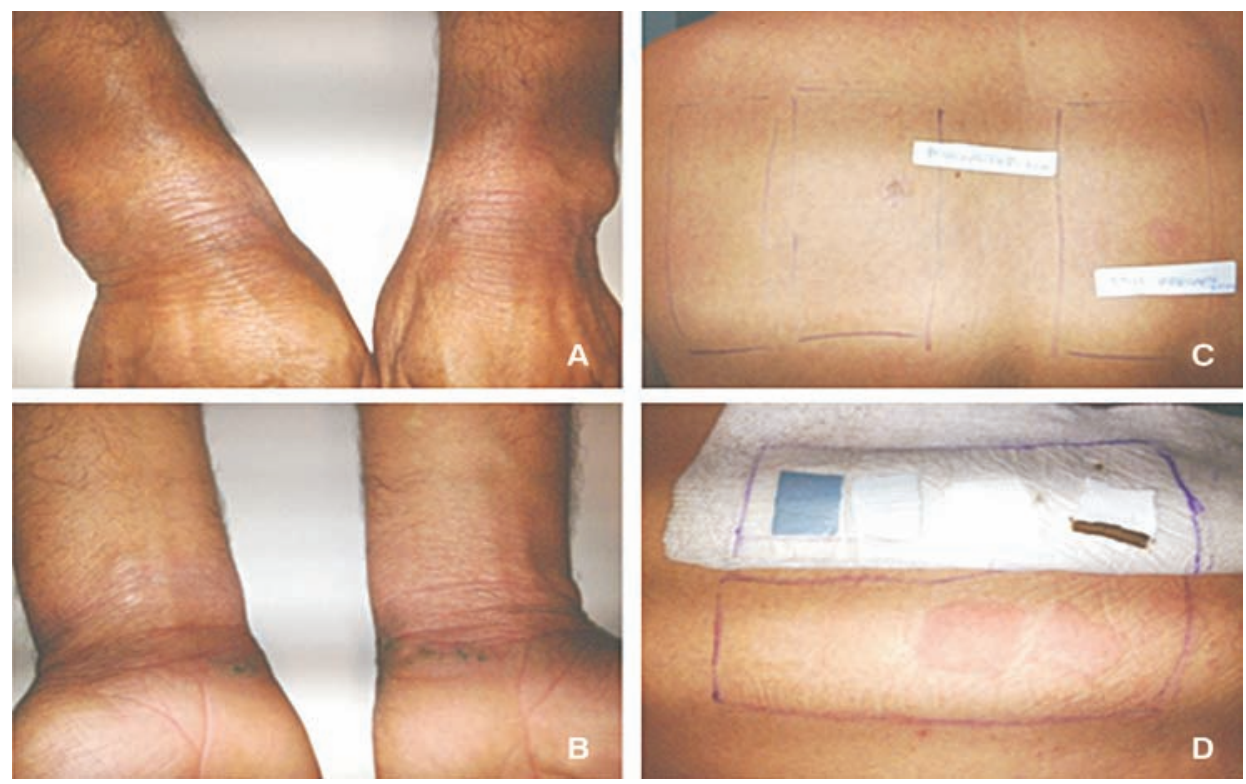

Fig 1 - Dermatite de contacto crónica na face dorsal (A) e palmar (B) de ambos os punhos. Leitura às 72h: mercaptobenzotiazol $(++)$ e mistura de mercapto $(++)(\mathbf{C})$ e leitura com os fragmentos da própria luva incluindo a faixa elástica presente no punho das luvas: $++(\mathrm{D})$.
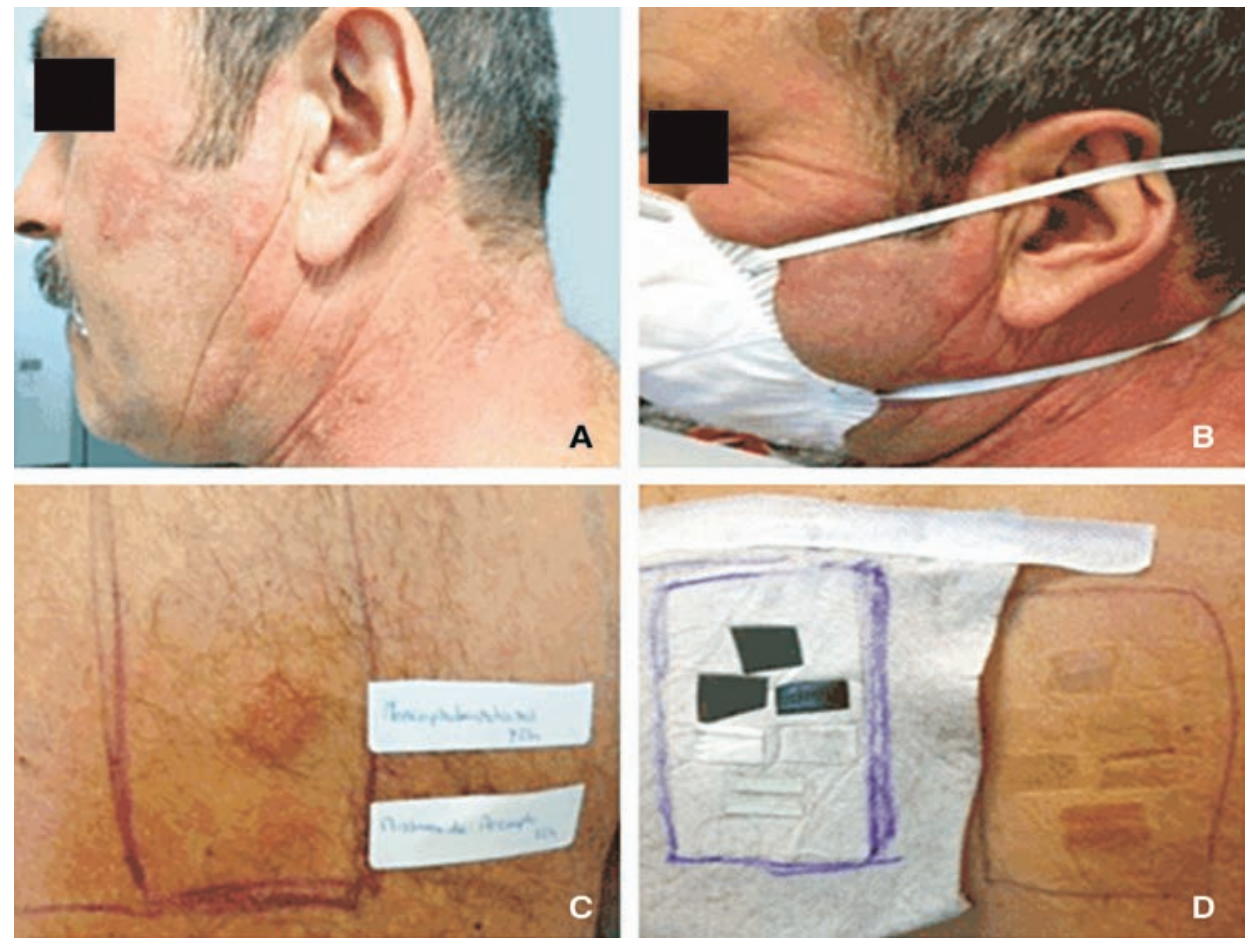

Fig 2 - Dermatite de contacto crónica na região geniana e cervical posterior (A); Máscara de proteção do local de trabalho (B); Leitura às $72 \mathrm{~h}$ : mercaptobenzotiazol $(++)$ e mistura de mercapto $(++) ;(C)$ leitura com os fragmentos da própria máscara incluindo a faixa elástica: ++ (D). 
realizada biópsia cutânea que demonstrou epiderme com acantose, hiperqueratose com focos de paraqueratose, exocitose linfocítica neutrofílica e presença de queratinócitos apoptóticos. Na derme superficial observa-se infiltrado inflamatório de predomínio perivascular no qual participam linfócitos, histiócitos e polimorfonucleares neutrófilos e eosinófilos. Foram realizados testes epicutâneos com a bateria padrão do Grupo Português de Estudo de Dermatite de Contacto, bateria de cosméticos e bateria de borrachas e colas. Fragmentos da máscara de proteção utilizada no local de trabalho e madeiras manipuladas pelo doente ( $20 \%$ em vaselina, em câmara fechada) também foram testados. Verificou-se positividade para mercaptobenzotiazol $(++)$, mistura mercapto $(++)$ e elástico de máscara $(++)$ na leitura das 72 horas e aos 7 dias (Fig.s 2c e 2d). Os restantes alérgenos testados, incluindo outras partes do corpo da máscara, foram negativos. Os testes Prick realizados com madeira foram negativos.

\section{DISCUSSÃO}

O mercaptobenzotiazol e a mistura de mercapto são alergénios bem conhecidos e a sua importância clínica como potenciais desencadeadores de dermatite de contacto alérgica (DCA) é evidente, ${ }^{4}$ estando incluídos na bateria padrão dos testes epicutâneos, do Grupo Português para o Estudo das Dermatites de Contacto. São aditivos químicos frequentemente utilizados na polimerização da borracha, como aceleradores de vulcanização, pelo que a lista de produtos de borracha que contém mercaptobenzotiazol é extensa ${ }^{1}$ (Tabela 1).

As fontes mais comuns de exposição e sensibilidade ao mercaptobenzotiazol são as luvas e os sapatos. As luvas têm sido descritas como as maiores causadoras de DCA em contexto ocupacional e a terceira causa mais comum de reações com teste epicutâneo positivo depois do calçado e dispositivos médicos ${ }^{5}$. As luvas de borracha sintética causam DCA por reação de hipersensibilidade retardada aos aditivos químicos da borracha utilizados na sua polimerização: em primeiro os tiurams, seguidos em ordem decrescente de frequência, os carbamatos, e mercaptos. O mercaptobenzotiazol é o segundo agente etiológico a seguir aos tiurams na DCA pelas luvas, enquanto que a dermatite dos pés é mais comumente causada pelo mercaptobenzotiazol e menos frequentemente devido aos tiurams ${ }^{5}$. Em exposição médica, os mercapto são causa comum de DCA pelas luvas, cateteres e adesivos elásticos. Além da indústria da borracha, são utilizados como fungicidas e anti-corrosivos na indústria do metal e na indústria mineira na colheita de ouro e prata.

A taxa de sensibilidade ao mercaptobenzotiazol nos testes epicutâneos varia entre os países6 de 1 a 8\%. A DCA ao mercaptobenzotiazol pode ser observada tanto em crianças quanto em adultos e parece ser mais frequente no sexo masculino, provavelmente devido ao maior uso de vestuário com aditivos da borracha ${ }^{7}$.

Os dados mais recentes na literatura descrevem

Tabela 1 - Lista de produtos que contém mercaptobenzotiazol e mistura de mercapto com fonte na borracha ou outras fontes.

Sapatos de borracha: solas, ténis

Sapatos de couro: adesivos, elásticos

Luvas: domésticas, trabalho, hospital

Vestuário, faixas elásticas

Preservativos

Dispositivos médicos: cateteres, adesivos, tiras de elétrodos de ECG

Brinquedos

Pneus, tubos, ligas, utensílios domésticos, tampões de ouvido, esponjas
Anticorrosivo

Cimento, moldes de cerâmica, Tintas, graxa

Detergentes, produtos de limpeza para sistemas de refrigeração de automóveis

Descongelante

Fungicida, microbicida

Medicamentos veterinários

Fotografia: emulsão do filme fotográfico 
uma relação entre a capacidade alérgica do mercaptobenzotiazol e seus compostos e a atividade ocupacional num terço dos doentes, ${ }^{8,9}$ embora possa ser variável consoante as séries de casos (como observado em apenas $1 \%$ dos casos numa revisão de 230 doentes com DCA ocupacional) ${ }^{10}$.

O baixo peso molecular do grupo mercaptobenzotiazol e a consequente permeabilidade cutânea e a existência do grupo tiol com capacidade de ligação a aminoácidos e proteínas são potenciais fatores que favorecem a sensibilização ${ }^{11-13}$. Contudo, a relativa baixa prevalência de alergia que se tem observado poderá favorecer um baixo potencial sensibilizante. Ainda assim, deve ser considerada importante a exposição ocupacional de doentes com reações positivas no teste epicutâneo ao mercaptobenzotiazol e compostos relacionados.

A dermatite de contacto alérgica à faixa elástica presente no punho das luvas e às faixas elásticas da máscara de proteção que se estendia da região geniana à região retro-auricular e cervical posterior, demonstram a importância da localização das lesões cutâneas quando se avalia uma dermatite de contacto, orientando o clínico para a suspeita mais provável. É de salientar que o mercaptobenzotiazol pode estar presente noutras fontes de exposição à borracha, tais como o tecido elástico no vestuário.

\section{CONCLUSÃO}

Apesar de serem alergénios bem conhecidos, o mercaptobenzotiazol e seus derivados continuam a ser largamente utilizados na indústria da borracha incluindo luvas, calçado, roupa, faixas elásticas, entre outros. É de realçar a relativa baixa prevalência de alergia, pelo que tem sido considerado ter baixo potencial sensibilizante. No entanto, uma vez o trabalhador sensibilizado, pode ser causa de incapacidade quer para a profissão quer para as atividades de vida diária.

\section{REFERÊNCIAS}

1. Adams A, Warshaw E. Allergic contact dermatitis from mercapto compounds. Dermatitis. 2006; 17(2):56-70.

2. Rudner EJ, Clendenning WE, Epstein E, et al. Epidemiology of Contact Dermatitis in North America:
1972. Arch Dermatol.1973; 108(4):537-40.

3. North American Contact Dermatitis Group. Interim report 2003- 2004. Chicago: North American Contact Dermatitis Group; 2005.

4. Diepgen T, Bruynzeel D, Andersen K, Brandão F, Bruze M, Gonçalo M, et al. Mercaptobenzothiazole or the mercapto-mix: which should be in standard series? Contact Dermatitis. 2006; 55(1):36-8.

5. Warshaw EM, Cook JW, Belsito DV, DeLeo VA, Fowler JF Jr, Maibach HI, et al. North American Contact Dermatitis Group. Positive patch-test reactions to mixed dialkyl thioureas: cross-sectional data from the North American Contact Dermatitis Group, 1994 to 2004. Dermatitis. 2008; 19(4):190-201.

6. Storrrs F, Rosenthal LE, Adams RM, Clendenning W, Emmett EA, Fisher AA, et al. Prevalence and relevance of allergic reactions in patients tested in North America. J Am Acad Dermatol. 1989; 20(6):103844.

7. Nethercott JR, Holness DL, Adams RM, Belsito D, Deleo V, Emmett EA, et al. Patch testing with a routine screening tray in North America, 1985 through 1989: II. Gender and response. Am J Contact Dermat. $1991 ; 2: 130-4$.

8. Rietschel RL, Mathias CGT, Fowler JF, Pratt M, Taylor $J S$, Sherertz EF, et al. Relationship of occupation to contact dermatitis: evaluation in patients tested from 1998 to 2000. Am J Contact Dermat. 2002; 13(4):170-6.

9. Dickel H, Kuss O, Schmidt A, Diepgen TL. Occupational relevance of positive standard patch-test results in employed persons with an initial report of an occupational skin disease. Int Arch Occup Environ Health 2002; 75(6):423-34.

10. Nettis E, Marcandrea MC, Paradiso MT, Paradiso MT, Ferrannini A, Tursi A. Results of standard series patch testing in patients with occupational allergic contact dermatitis. Allergy. 2003; 58(12): 1304-7.

11. Belsito DV. The immunologic basis of patch testing. J Am Acad Dermatol. 1989; 21 (4 Pt 2): 822-9.

12. Rietschel RL, Fowler JF .The pathogenesis of allergic contact hypersensitivity. 5th ed, Philadelphia: Lippincott Williams \& Wilkins; 2001.

13. Wang $X$, Tabor MW. Studies of the reactivity of morpholine, 2-mercaptobenzothiazole and 2 of their derivatives. Contact Dermatitis. 1988; 19(1):1621. 